\title{
A New Method for the Measurement of
}

\section{Thyroidal Iodine Release in Man}

\author{
JoHN T. NicolofF \\ From the University of Southern California School of Medicine and the Los \\ Angeles County-University of Southern California Medical Center, \\ Los Angeles, California 90033
}

\begin{abstract}
A B S T RACT A new method is described for the detection of variations in the release rate of all iodinated products by the thyroid gland in man. ${ }^{125} \mathrm{I}$ was employed to endogenously label the thyroid gland, and a parenterally administered ${ }^{181} \mathrm{I}$ thyroxine dose was used to generate a ${ }^{181} \mathrm{I}$ reference source. Thyroidal iodine release activity was quantitatively assessed by the measurement of variations in ${ }^{125} \mathrm{I} /{ }^{181} \mathrm{I}$ ratio values obtained in timed urine samples. Diurnal variation in thyroid release patterns was observed in euthyroid subjects which was promptly suppressed by exogenous triiodothyronine administration and was simulated by the intramuscular injection of $0.25-0.50 \mathrm{U}$ of bovine thyrotropin. The zenith value occurred at 4:00 a.m. \pm 3.4 $\mathrm{hr}$ (SD) and the nadir at 5:00 p.m. $\pm 3.6 \mathrm{hr}$. The absence of a diurnal pattern in patients with thyrotoxicosis and in secondary hypothyroidism indicated that this diurnal fluctuation was under thyrotropin (TSH) regulation. This new method also promises to be a useful tool for the study of the intrathyroidal recycling of iodide from the iodotyrosine pool and the detection of factors which may acutely alter thyroid function.
\end{abstract}

\section{INTRODUCTION}

The thyroid gland possesses a large reservoir of preformed hormone which enables it to meet sudden demands for increased hormone secretion (1). Studies of thyroid function performed in laboratory animals have revealed that this reserve capacity is frequently tested by a wide variety of both physiological and pharmacological stimuli (2-9). In contrast, similar studies performed in man have not revealed such lability in thyroid function (10-15). Indeed, the phylogenetic development in man of a large extrathyroidal pool of thyroxine bound to thyroxine-binding proteins would appear to

Received for publication 22 April 1970 and in revised form 18 June 1970. act to assure constancy of this supply of thyroxine to peripheral target tissues by buffering variations in hormone secretion. These observations raised the questions of whether control mechanisms for regulation of thyroid hormone release in man function to assure maximum resistance to change or whether the previous methods employed for the detection of such changes have been too insensitive.

As an outgrowth of recent studies conducted in our laboratory regarding the measurement of thyroxine deiodination rate (16), a new technique was developed for the detection of acute alterations in the rate of thyroidal iodine release. This study details the basic method employed in this technique as well as the general areas for its application in the study of both normal and abnormal thyroid function states.

\section{METHODS}

Principles of methodology. The proposed method was designed to qualitatively measure in vivo variations in the release rate of iodine (as $\mathrm{I}^{-}$and iodinated products) from the thyroid gland prelabeled with ${ }^{125} \mathrm{I}$. The urine was selected as the site for measurement, not only because it reflected all iodinated release products but also because it provided an integrated time measurement during the interval of urine formation. Normally, iodide $\left(I^{-}\right)$, triiodothyronine $\left(T_{3}\right)$, and thyroxine $\left(T_{4}\right)$ comprise the majority of the iodinated products released by the thyroid gland. The ${ }^{125} \mathrm{I}$ released directly by the thyroid may be cleared into the urine while the $T_{3^{-}}{ }^{125} \mathrm{I}$ and the $\mathrm{T}_{4}{ }^{-125} \mathrm{I}$, because of their binding to circulating proteins, are virtually excluded from the urine and must first be deiodinated by the peripheral tissues before the ${ }^{125}$ I can be excreted. Because the renal and extrarenal clearances of $\mathrm{I}^{-}$may vary spontaneously, a ${ }^{181} \mathrm{I}$ constant reference source was introduced into the system to negate this variable. The ${ }^{181} \mathrm{I}$ could have been provided as a constant infusion as had been employed in our previous studies of $\mathrm{T}_{4}$ peripheral metabolism (16), but due to the regularity of $T_{4}$ deiodination in vivo, $T_{4^{-181}} \mathrm{I}$ itself was used. In a sense, the deiodination of $\mathrm{T}_{4}{ }^{131} \mathrm{I}$ served as a type of biological constant infusion system. Comparison of the ratio values of urinary ${ }^{125} \mathrm{I}$ derived from the thyroid gland to the ${ }^{181} \mathrm{I}$ gen- 
erated by the $T_{4^{-181}}{ }^{18} \mathrm{I}$ reference source reflected variations in the rate of thyroidal iodine release. This ${ }^{125} \mathrm{I} /{ }^{181} \mathrm{I}$ ratio analysis had the technical advantage of not requiring complete recovery of the $\mathrm{I}^{-}$isotopes; and since $\mathrm{T}_{4}$ comprised one of the major thyroid release products, its use as a reference source also rendered the interpretation of the urinary ${ }^{125} \mathrm{I} /{ }^{181} \mathrm{I}$ ratio data more meaningful.

Isotopic labeling technique. Endogenous labeling of the thyroid gland was accomplished by the oral administration of a carrier-free tracer dose of sodium- ${ }^{125}$ I. On the basis of a prior thyroid- ${ }^{131} \mathrm{I}$ uptake value, a dose was selected to deliver approximately $10 \mu \mathrm{Ci}$ of ${ }^{125} \mathrm{I}$ to the thyroid gland. At the same time, $25 \mu \mathrm{Ci}$ of $3^{\prime}, 5^{\prime}-{ }^{131}$ I-labeled thyroxine was given intravenously. Specific activity of the $\mathrm{T}_{4^{-181}} \mathrm{I}$ tracer at the time of administration was greater than $20 \mu \mathrm{Ci} / \mu \mathrm{g}$. These isotopes were obtained commercially from Abbott Laboratories, North Chicago, Ill or from Industrial Nuclear Corporation, St. Louis, Mo. The $\mathrm{T}_{\mathbf{4}^{-}}{ }^{181} \mathrm{I}$ tracer was prepared for intravenous administration by dilution in a sterile normal saline solution containing $1 \%$ human serum albumin. No antithyroid drugs or blocking agents were used during the course of the studies unless expressly stated in the text.

Patients employed in this study were from the inpatient and outpatient services of the Los Angeles County-University of Southern California Medical Center and were studied in the Clinical Research Center of that institution. Diagnosis of the thyroid status of each patient was determined by clinical evaluation and was supported by appropriate conventional laboratory tests for thyroid function.

Collection procedure. After allowing a 2 day interval for the labeled thyroxine to approach equilibration in the extrathyroidal $T_{4}$ pool (17), urinary sampling was begun. Timed urine collections were obtained in approximately 2-hr intervals during the waking hours and intermittently throughout the night when subjects awoke spontaneously. The clock time assigned to each sample was the time when the urine sample was voided. The samples were collected in urine specimen bottles into which 3 cc of IRA 400 anion exchange resin, chloride cycle, 20-50 mesh (Mallinckrodt Chemical Works, St. Louis, Mo.) had been added. The urine samples were then allowed to incubate with the resin for a period of at least $24 \mathrm{hr}$ at room temperature in order to facilitate the uptake of the labeled $\mathrm{I}^{-}$on the resin granules. Samples were then decanted and the resin was transferred to glass counting tubes. The samples were then counted for both ${ }^{125} \mathrm{I}$ and ${ }^{181} \mathrm{I}$ activity in an automatic welltype scintillation counter employing a dual channel spectrophotometer (Baird-Atomic, Inc., Cambridge, Mass.). Each sample was counted until the accumulated net counts for each isotope exceeded 2500 . The results were expressed as ratio values of the ${ }^{125} \mathrm{I} /{ }^{181} \mathrm{I}$ plotted on semilogarithmic coordinates against time in days. The day marker on all graphs represented midnight.

\section{RESULTS}

The pattern of thyroid release in euthyroid subjects. A representative thyroidal iodine relase pattern in a euthyroid subject is illustrated in Fig. 1. For the purpose of analysis, this pattern was divided into three phases. These comprised excretion, transition, and release slope phases.

During the excretion phase, the orally administered ${ }^{125} \mathrm{I}$, which had been employed to exogenously label the thyroid gland, had not yet been dissipated from the extrathyroidal $\mathrm{I}^{-}$pool. This was reflected by the rapid exponentially linear decline in the urinary ${ }^{125} \mathrm{I} /{ }^{131} \mathrm{I}$ ratio slope values generated during this phase. The half-life of this slope approximated $8-10 \mathrm{hr}$ which was similar to that of the biological half-life of $\mathrm{I}^{-}$in man. The transition phase was marked by the juncture where the ${ }^{125} \mathrm{I} /{ }^{181} \mathrm{I}$ urinary ratio slope values departed from linearity. This occurred shortly after the beginning of the 3rd day.

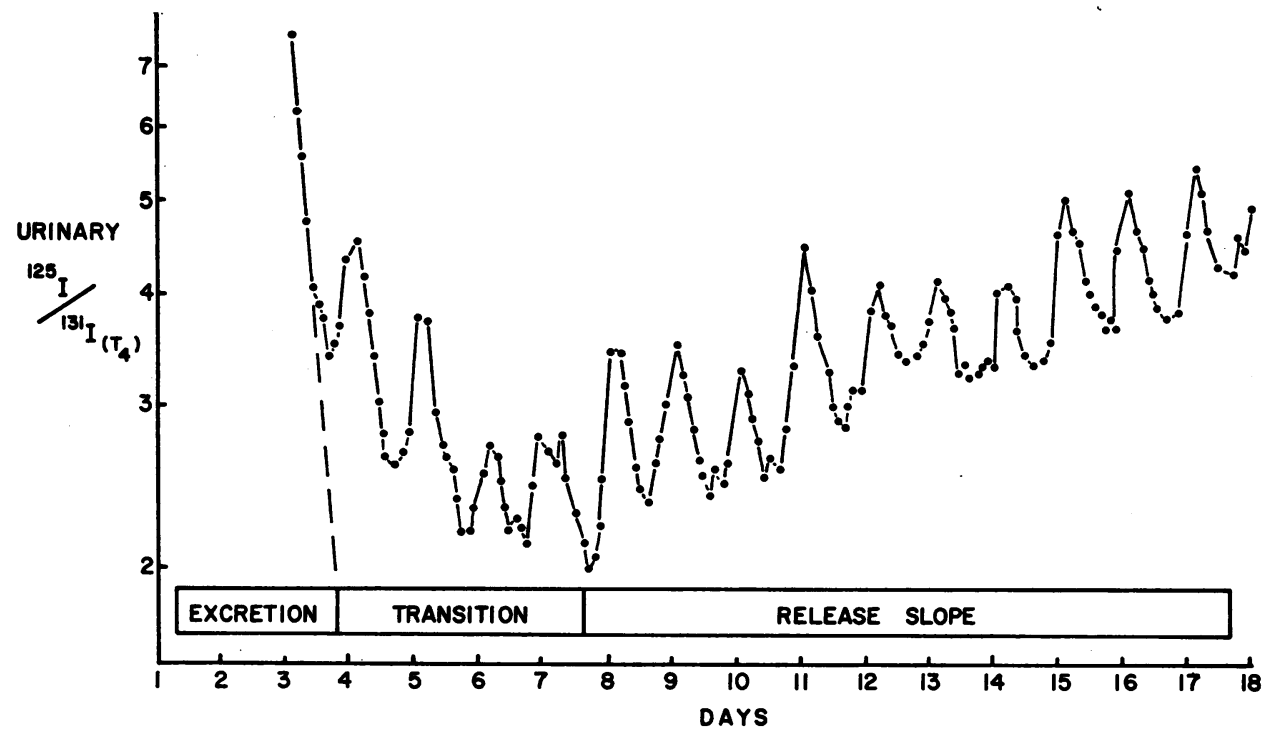

Figure 1 Normal control. A typical thyroid release study in a euthyroid subject. The pattern is divided into excretion, transition, and release slope phases. The dotted line represents an extrapolation of the original slope developed during the excretory phase. 
Projection of the initial linear slope is depicted as an interrupted line in Fig. 1. The rapid drop of the slope indicated that the ${ }^{125} \mathrm{I}$ originally employed to label the thyroid gland played only a minor role in determining the ${ }^{125} \mathrm{I} /{ }^{131} \mathrm{I}$ urinary ratio values observed during the transition phase. Over the subsequent 4 days a gradual and nonlinear decline was seen in the urinary ratio values. This decline was punctuated daily by a rapid rise and fall in values. Termination of the transition phase occurred about 1 wk after the initiation of the study. The beginning of the release slope phase was marked by a gradual linear logarithmic rise in the ratio values. This linearity was observed to be maintained in this subject for a $1 \frac{1}{2}$ wk period. In other studies of euthyroid subjects, this linearity had been found to be maintained for periods as long as $4 \mathrm{wk}$. Again, as in the transition phase, rapid daily fluctuations in the ratio values were observed.

The pattern of diurnal variations in thyroidal iodine release in euthyroid subjects. To date 76 euthyroid subjects have been studied employing this technique. The ages of these subjects ranged from 16 to $74 \mathrm{yr}$ and the sex distribution was 35 females and 41 males. Without exception, these euthyroid subjects exhibited a diurnal variation in thyroid iodine release during the transition and release slope phases. The timing of these daily cycles varied somewhat from subject to subject. The zenith value occurred anywhere from 8:00 p.m. to 12:00 noon, with the mean value being 4:00 a.m. \pm 3.4 $\mathrm{hr}$ ( $\pm \mathrm{SD})$. The nadir value was 5:00 p.m. $\pm 3.6 \mathrm{hr}$. A histogram of the zenith and the nadir values for the 76 euthyroid subjects is illustrated in Fig. 2. One notable case was a subject who had a reverse sleep-wake pattern for a period of greater than $15 \mathrm{yr}$. His peak release value occurred at 12:00 noon and his nadir value at 2:00 a.m. This reversal of the diurnal thyroid release pattern was also paralleled by a reversal in diurnal plasma cortisol values. Although there was considerable variation in the peak and nadir values occurring in different subjects, the individual patterns were remarkably reproducible. This is illustrated in Fig. 3 where comparison is made of three consecutive release studies performed in a single euthyroid subject.

The effect of various thyroid release products on the ${ }^{125} I /{ }^{131} I$ urinary ratio values. In an effort to gain a better understanding of the complex character of the spontaneous ${ }^{125} \mathrm{I} /{ }^{131} \mathrm{I}$ thyroid release pattern, a study was performed in which an attempt was made to simulate the release patterns produced by each of the major iodinated products of the thyroid gland. Equal isotopic doses of ${ }^{125} \mathrm{I}^{-}, \mathrm{T}_{3}{ }^{125} \mathrm{I}$, and $\mathrm{T}_{4}{ }^{-225} \mathrm{I}$ were given intravenously as pulse injections in a euthyroid subject in whom endogenous release had been suppressed by exogenous, stable $T_{3}$. The results of this study are illustrated in Fig. 4. For purposes of comparison, the data have been normalized so that zero time represented the time of injection. The initial urinary sample after the acute injection of ${ }^{125} \mathrm{I}$ produced the highest ${ }^{125} \mathrm{I} /{ }^{181} \mathrm{I}$ ratio value. Subsequently, the ratio values fell rapidly until it assumed the original base line value in approximately $20 \mathrm{hr}$. After a similar injection of $\mathrm{T}_{3^{-1}}{ }^{25} \mathrm{I}$, there was a prompt rise in urinary ${ }^{125} \mathrm{I} /{ }^{181} \mathrm{I}$ ratio values which plateaued at $20 \mathrm{hr}$. This was followed by a slower decline to the base line values over the next $50 \mathrm{hr}$. After the injection of $T_{4}{ }^{125} \mathrm{I}$, there was a very slow rise in ratio values which did not plateau until $60 \mathrm{hr}$. Subsequently, the ratio values remained stationary and permanently elevated above the base line. The reason for the very slow

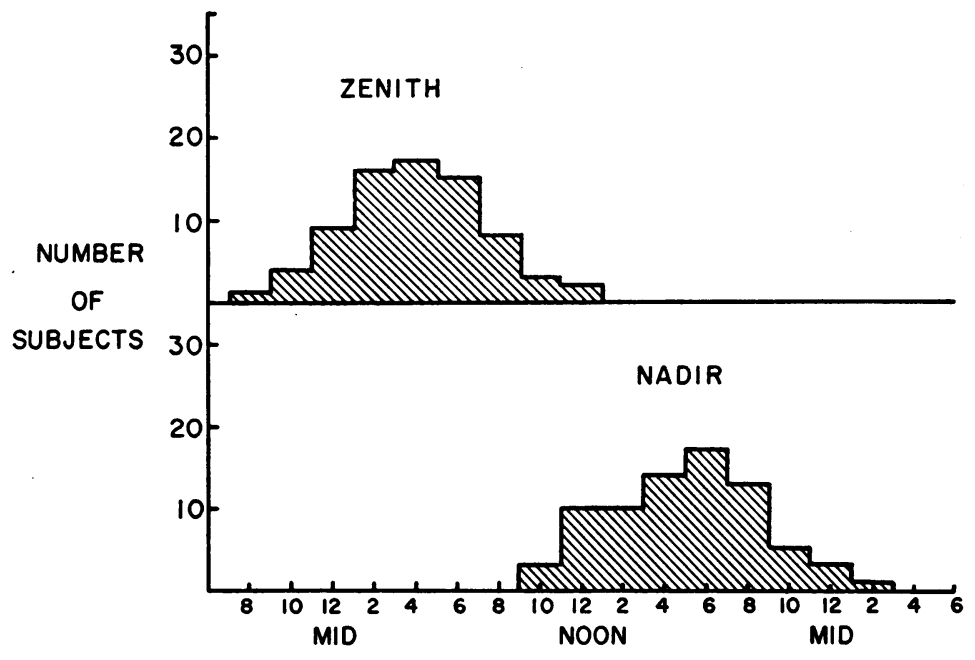

Figure 2 A histogram of the average zenith and nadir values of diurnal thyroid release patterns observed in 76 euthyroid subjects. 
rise in ratio values after $T_{-1}{ }^{125} \mathrm{I}$ was because of the long equilibration period required for this hormone in the extrathyroid body pools (17). The reason the ultimate ratio value became fixed and did not drop was because $\mathrm{T}_{4}{ }^{181} \mathrm{I}$ served as the reference isotope. This experiment (as shown in Fig. 4) has been conducted in two other subjects with similar results.

These observations indicated that the endogenous release of ${ }^{125} \mathrm{I}$ - and $\mathrm{T}_{3}{ }^{-125} \mathrm{I}$ would have resulted in a prompt and relatively large change in urinary ratio values. This was especially true of ${ }^{125} \mathrm{I}^{-}$. In contrast, isotopically equal amounts of $T_{4}{ }^{205} \mathrm{I}$ resulted in a less dramatic but a permanent increase in the ${ }^{125} \mathrm{I} /{ }^{131} \mathrm{I}$ ratio values. It was apparent that the diurnal fluctuation in ${ }^{125} \mathrm{I} /{ }^{131} \mathrm{I}$ urinary ratio values observed in euthyroid subjects must have been primarily accounted for by the variation in the release of ${ }^{125} \mathrm{I}^{-}$by the thyroid gland and could not have been a reflection of variations of $T_{4}{ }^{125} \mathrm{I}$ release. The release of endogenously labeled mono-or iodotyrosine will produce a release pattern indistinguishable from that of $\mathrm{I}^{-}$since they share a similar biological half-life (18).

Regulation of thyroidal iodine release. Normally thyrotropin (TSH) regulates the release of thyroid hormone by the thyroid gland (1). Therefore, the administration of exogenous thyroid hormone, acting via a negative feedback mechanism to inhibit TSH release, should cause a prompt inhibition of thyroid glandular release. The effect of exogenously administered $\mathrm{T}_{3}$ and bovine TSH on thyroid release is illustrated in Fig. 5. A prompt inhibition of release was noted in euthyroid subject $\mathrm{A}$

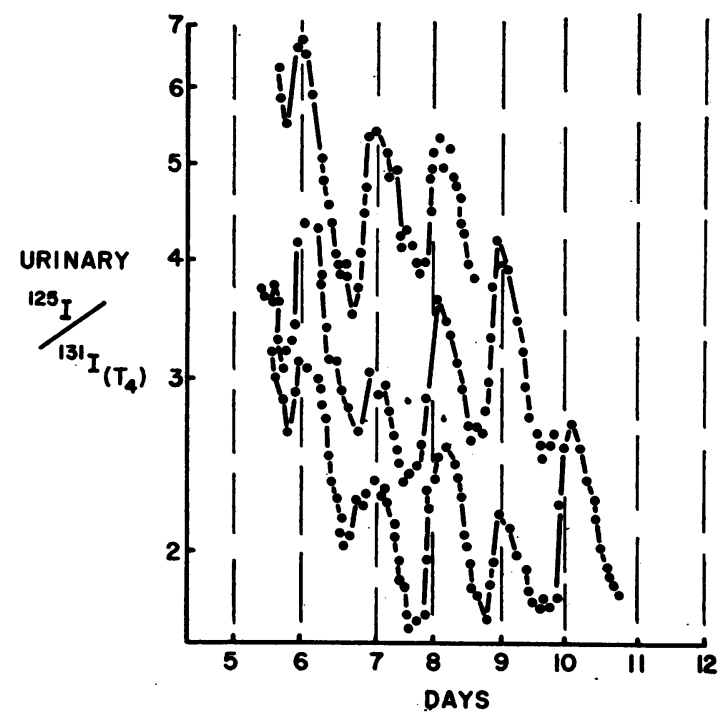

FIGURE 3 Thyroid release patterns observed in a single euthyroid subject in three separate studies. The vertical broken lines are midnight reference markers. Note that the daily peak and nadir values and the general form of the release patterns were similar in all three studies.

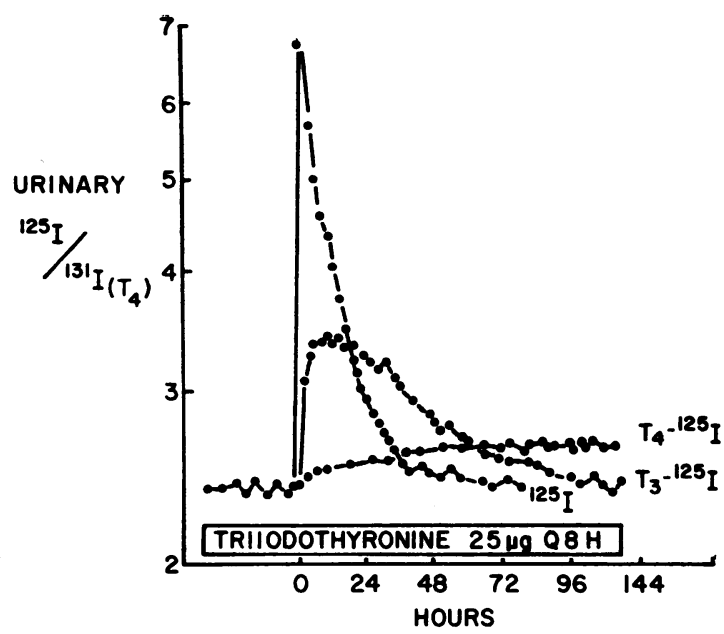

FIGURE 4 A simulation of the effects of each of the major thyroid release products on urinary ${ }^{125} \mathrm{I} /{ }^{181} \mathrm{I}$ ratio values. Equal isotopic doses of $\mathrm{T}_{4}{ }^{125} \mathrm{I}$ and $\mathrm{T}_{3^{-}}{ }^{125} \mathrm{I}$ and ${ }^{125} \mathrm{I}^{-}$were used. Endogenous release was suppressed by triiodothyronine administration.

with the initial $25 \mu \mathrm{g}$ oral $\mathrm{T}_{\mathbf{3}}$ dose. Similar acute inhibition could be observed with $T_{4}$, but only at much larger dosage levels. While under $T_{s}$ suppression, the intramuscular administration of bovine TSH in dosages ranging between 0.25 and $1.0 \mathrm{U}$ resulted in a similar release pattern to that produced by spontaneous diurnal release cycles. In contrast, subject $B$, a patient with hypothyroidism secondary to hypopituitarism, exhibited no spontaneous diurnal patterns of thyroid release. The intramuscular administration of TSH again produced a prompt release response. Note that the release response was proportional to the dose of TSH administered. The limit of sensitivity appeared to be approximately equal to the stimulatory equivalent of $0.25 \mathrm{U}$ of bovine TSH. Subject $\mathrm{C}$ demonstrated the pattern of thyroid release found in a typical patient with toxic Graves' disease. As in the case of secondary hypothyroidism, there was no evidence of a diurnal pattern. In contrast to euthyroid subjects, no inhibition in thyroid release was produced with exogenous $T_{3}$ administration, but the injection of bovine TSH produced a prompt augmentation in glandular release. Subjects with toxic nodular goiters produced a similar pattern to that of Graves' disease. Subject D illustrated the pattern of release in a patient with primary hypothyroidism. As in the case of secondary hypothyroidism and Graves' disease, a loss in diurnal pattern was noted. But in contrast to those subjects, exogenous TSH failed to augment release. To date we have studied 4 patients with primary hypothyroidism, 16 patients with secondary hypothyroidism, and 44 patients with toxic Graves' disease employing this release technique. The findings illustrated in Fig. 5 were typical of patients studied with these disease conditions. 


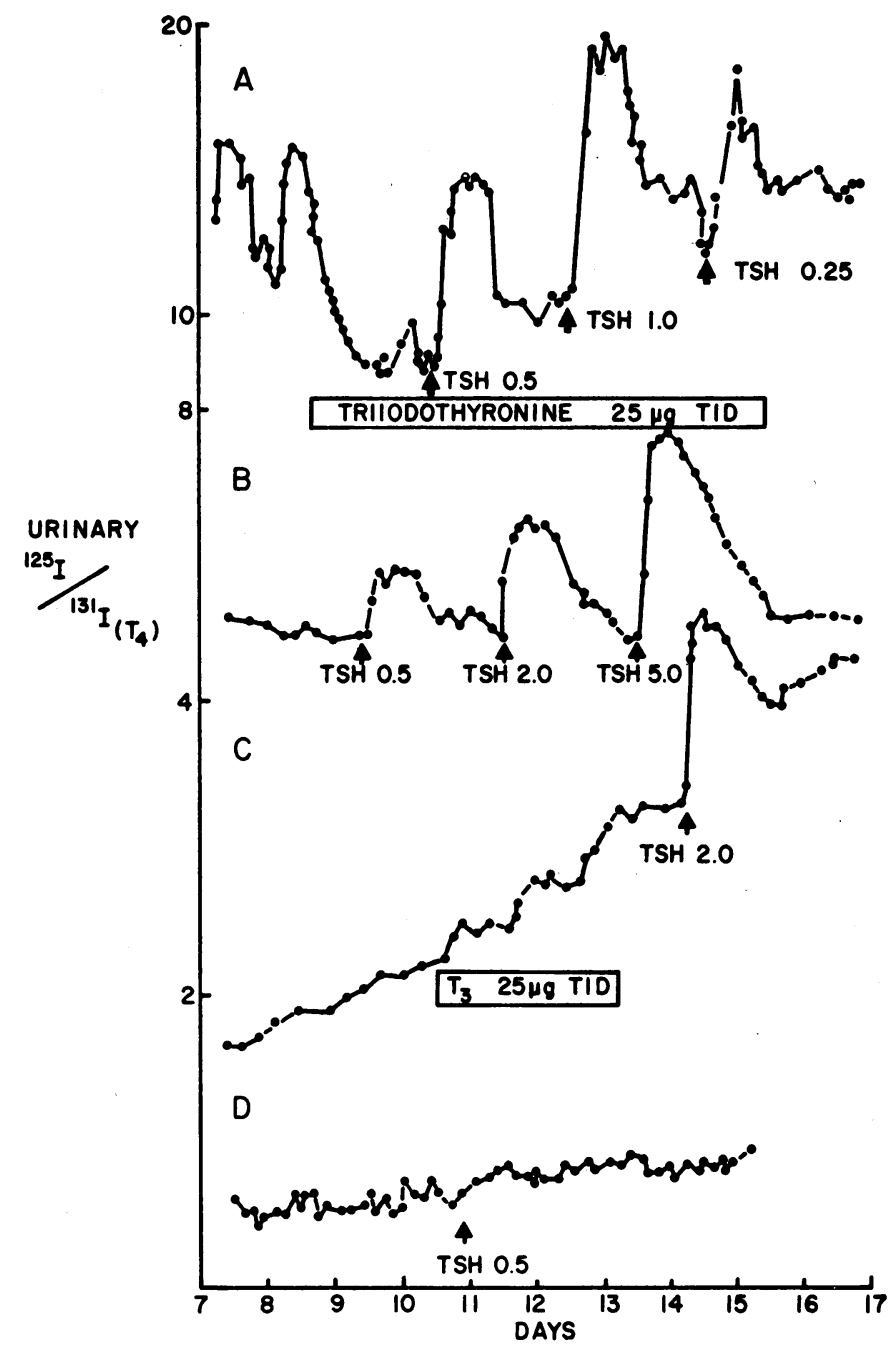

FIGURE 5 Control release pattern and the effects of intramuscular bovine TSH injections in a euthyroid (A), a secondary hypothyroid (B), a toxic Graves' disease (C), and a primary hypothyroid (D) subject.

It should be noted that the rate of rise in ratio values during the release phase in the typical thyrotoxic subject was greater than that found in the euthyroid patient and, in turn, more than that observed in the hypothyroid patient. Additionally, the release slope phase was reached much sooner in the hyperthyroid patient than in the euthyroid and hypothyroid subjects. These differences were presumably due to the amount and rate at which the ${ }^{125} \mathrm{I}$-labeled hormonal stores were being mobilized. The abrupt outpouring of ${ }^{125} \mathrm{I}$ from the thyroid gland after TSH stimulation, primarily represented a marked augmentation in the release of $\mathrm{I}^{-}$from the thyroid in addition to hormonal release. This augmentation in the $\mathrm{I}^{-}$leak from the thyroid gland stimulated with $\mathrm{TSH}$ has been previously observed in laboratory ani- mals (19). This view is supported by the observation that there was a rapid return of the ratio values to base line as would be expected if $\mathrm{I}^{-}$had been the predominating release product.

Studies of the second I pool. There has been an accumulating body of evidence both in man and in animals which indicates that there are two functional $\mathrm{I}^{-}$pools which serve as sources for new hormone formation (20-24). These pools are generally referred to as the first and second pool. The first $\mathrm{I}^{-}$pool represents $\mathrm{I}^{-}$held by the thyroid trapping mechanism which is freely exchangeable with the extrathyroidal $\mathrm{I}^{-}$. This first $\mathrm{I}^{-}$pool is derived from the peripheral tissue deiodination of $T_{3}$ and $T_{4}$ as well as from dietary sources. The second $\mathrm{I}^{-}$pool is created from the intrathyroidal deiodination of iodoty- 
rosines which are by-products of thyroglobulin hydrolysis. A large portion of this $\mathrm{I}^{-}$is thought to be recycled into new hormone formation without leaving the confines of the thyroid gland or exchanging with the first $\mathrm{I}^{-}$pool. Although some exchange most likely does occur between the first and second $\mathrm{I}^{-}$pools (25) under certain conditions, these two pools normally would appear to exhibit a marked degree of functional independence. This method provides an opportunity to measure the functional state of the second $\mathrm{I}^{-}$pool divorced from the first $\mathrm{I}^{-}$pool.

Fig. 6 illustrates the effects of three antithyroid drugs on thyroid release patterns in euthyroid subjects. When methimazole (Tapazole) was given to subject $A$, there was a prompt and sustained rise in the ${ }^{20} \mathrm{I} /{ }^{21} \mathrm{I}$ ratio values in the urine. This rise was apparently due to a block in new hormone formation in the face of continued hormone secretion. In other words, the $\mathrm{I}^{-}$coming

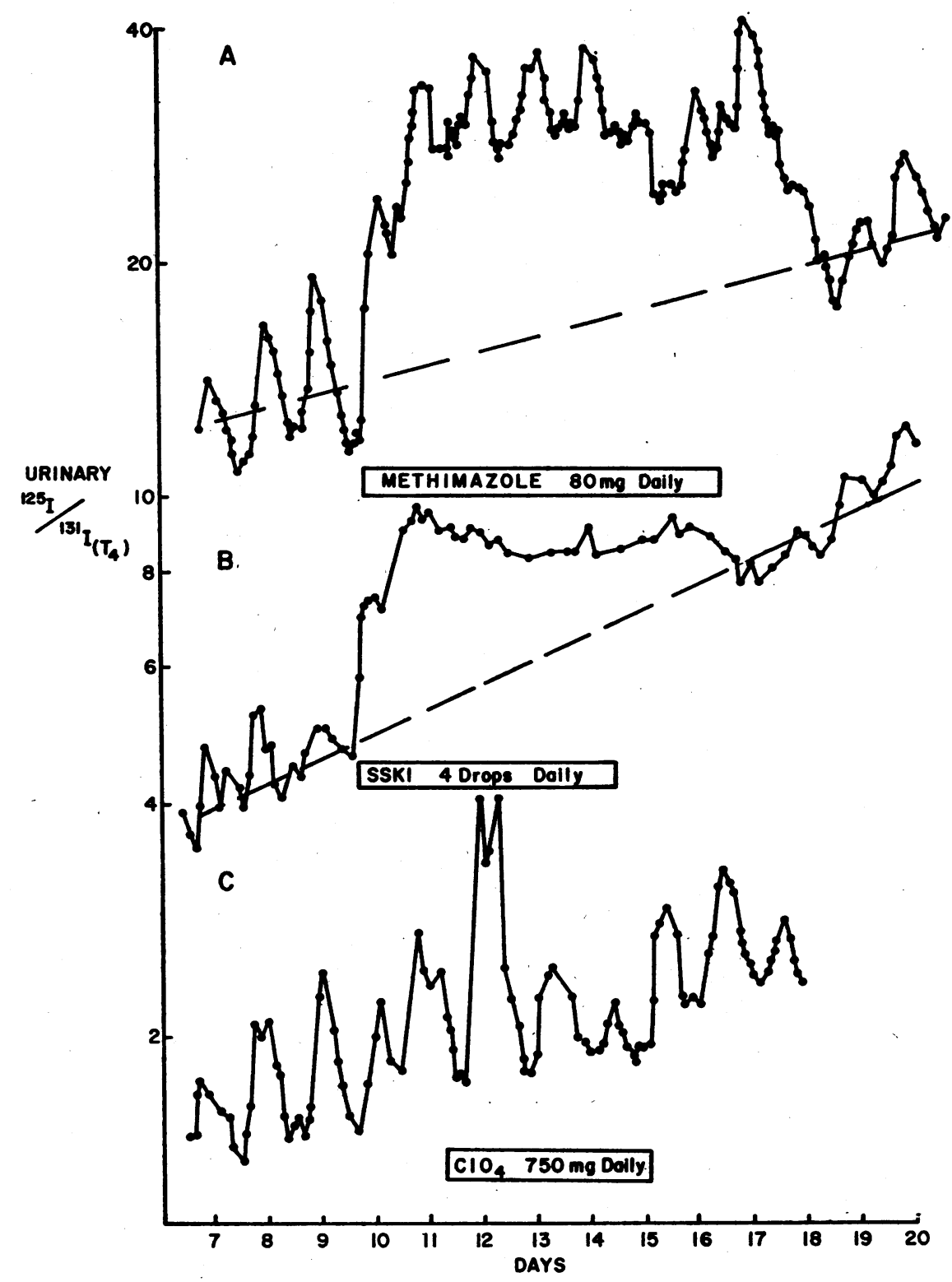

FIGURE 6 Effects of methimazole, potassium iodide, and perchlorate on thyroid release patterns in three euthyroid subjects. The interrupted line represents the projected normal slope of thyroid iodine release had no antithyroid drug been administered. 
into the second $\mathrm{I}^{-}$pool from iodotyrosine sources could not be incorporated into newly formed thyroglobulin and after exceeding the reservoir of capacity of the second $\mathrm{I}^{-}$pool, the $\mathrm{I}^{-}$leaked out of the thyroid gland. The fall in ratio values, 1 day after discontinuance of methimazole, was similar to that seen for $\mathrm{I}^{-}$as previously demonstrated in Fig. 4. This observation served to validate the interpretation of an $\mathrm{I}^{-}$leak from the second $\mathrm{I}^{-}$pool. The $24 \mathrm{hr}$ interval between the discontinuance of methimazole and the beginning of the fall of ratio values may be a reflection of the duration of the blocking action of this drug. In subject B a similar augmentation in ratio values was seen when large amounts of $\mathrm{KI}$ were administered. But in contrast to the action of methimazole, the $\mathrm{I}^{-}$leak was not sustained as indicated by a fall in the ratio values toward the projected normal release slope (interrupted line in Fig. 6). It has been previously demonstrated that $\mathrm{I}^{-}$ in large amounts is capable of producing a transient inhibition of organification by the so-called "WolffChaikoff" effect $(24,26)$. These findings are consistent with the known action of $\mathrm{I}^{-}$in this regard. These changes in ratio values, produced after methimazole and potassium $\mathrm{I}^{-}$administration, were not due to changes in the deiodination rate of the reference thyroxine isotope (16). Subject $C$ illustrated the effect of perchlorate administration on thyroid release. No significant change in urinary $\mathrm{I}^{-}$release pattern was observed in this patient or in four other patients treated in a similar manner.

The reference ${ }^{181} \mathrm{I}$ isotope used in this technique effectively eliminated the measurement of any alteration in the functional state of the first $\mathrm{I}^{-}$pool. If there was free exchange between the inner and the outer $\mathrm{I}^{-}$pools in these subjects, interference with organification processes by antithyroid drugs would not have resulted in a change in urinary ${ }^{125} \mathrm{I} /{ }^{121} \mathrm{I}$ ratio values. Since a marked increase was observed, this indicated that a functional separation between the two pools does exist in euthyroid subjects. Perchlorate, since it competed only with the $\mathrm{I}^{-}$in the first pool, produced no change in thyroid release patterns.

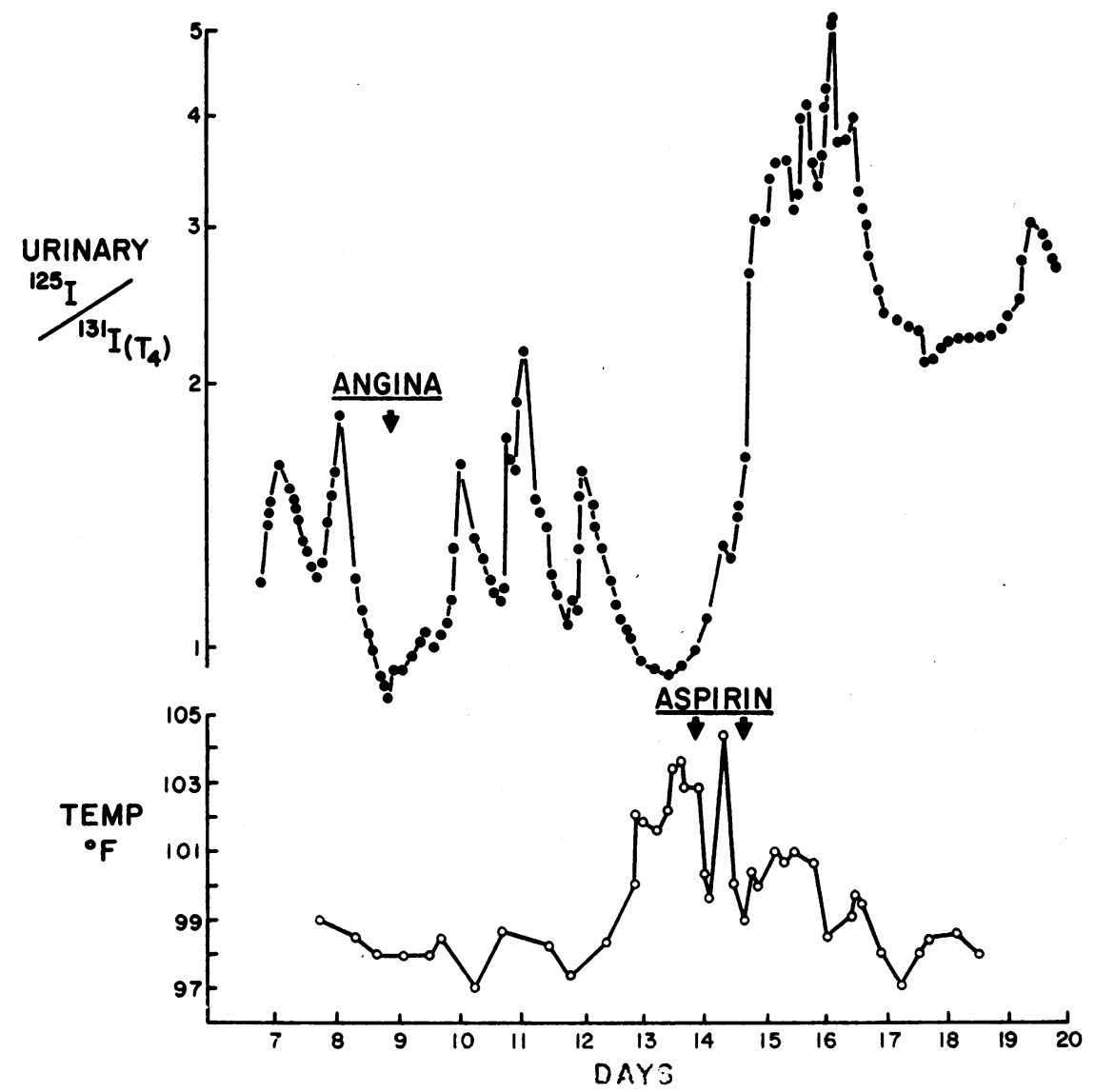

Figure 7 Spontaneous alterations in thyroid release patterns coincident with an attack of angina pectoris and Asian influenza. 
Environmental factors which may influence thyroidal iodine release. Generally, euthyroid subjects exhibited a diurnal pattern of thyroidal iodine release which was consistent in its timing and magnitude, but occasionally this regular pattern was spontaneously interrupted. Such an interruption in the normal pattern of thyroidal iodine release is illustrated in Fig. 7. This patient displayed a uniform iodine release cycle until he suffered a bout of anginal chest pain. The patient complained of severe pain and received nitroglycerine sublingual as noted by the arrow in the illustration. Even before this complaint had been registered with the nurse, suppression of the normal diurnal release cycle was evident. In a similar manner, when the patient developed what was to be diagnosed as Asian influenza on the 13th day, the thyroidal iodine release pattern appeared to be suppressed before a significant temperature elevation had taken place. When temperatures were rising, suppression of the release slope occurred. When the temperature curve began to spontaneously decline, a surge in thyroid activity was seen. This surge appeared to be of a compensatory nature indicating that a significant deficit in circulating thyroid hormone levels had occurred during the interval of suppressed thyroidal iodine release.

\section{DISCUSSION}

The thyroidal iodine release method, which has been described in this study, appears to be a sensitive tool for the measurement of fluctuations in the release rate of all iodinated products of the thyroid gland. The daily fluctuations in thyroidal iodine release are accountable almost entirely by variations in the $\mathrm{I}^{-}$leak from the thyroid gland. Under normal conditions, the magnitude and timing of the $\mathrm{I}^{-}$leak serves as a sensitive indicator of the state of thyroid glandular stimulation (19). The technique is primarily qualitative although some semiquantitative interpretation may be made by the exogenous administration of known amounts of TSH. The use of an I reference indicator, generated from the deiodination of $T_{4}{ }^{201} I$, effectively removed the interference of variations in the renal and/or thyroid $\mathrm{I}^{-}$ clearance as well as obviating the need for complete isotopic recoveries. The type and proportion of iodinated products released from the thyroid gland can sometimes be ascertained when employing an agent whose administration can be controlled. For example, methimazole was noted to promptly augment urinary ${ }^{105} \mathrm{I} /{ }^{181} \mathrm{I}$ ratio values. It can be reasoned that this augmentation was primarily due to an increase in the $\mathrm{I}^{-}$leak from the second $\mathrm{I}^{-}$pool because of the rapidity . with which the values were observed to drop after drug withdrawal. If $T_{3}$ release had been the primary cause of the augmentation, the fall in values after the drug withdrawal would have been much slower; and if $T$, had been the exclusive release product, no fall would have occurred at all. Thus, some gross indication of the type and character of thyroid release products can be made under certain circumstances.

Although analysis of the falloff in ratio slope values may give some insight into the nature of the labeled release products $\left(I^{-}, T_{3}\right.$, and $\left.T_{4}\right)$ from the thyroid gland, this information cannot be translated into terms of stable iodine counterpart. In order to interpret the fall in the ratio slope, the specific activities of all of these release products must be determined both as they are being secreted by the thyroid gland and in their respective extrathyroidal pools. In other words, the measurement of $T_{4}$ specific activity, which is technically quite simple, does not give any insight into the specific activities of $T_{3}$ and $I^{-}$. For example, the large fluctuations in $\mathrm{I}^{-}$release result from a leak in the intrathyroidal $\mathrm{I}^{-}$pool. The specific activity of intrathyroidal $\mathrm{I}^{-}$pool probably bears little relationship to the specific activity of $\mathrm{I}^{-}$in the extrathyroidal pool. Thus, it would appear difficult, if not impossible, to interpret urinary ratio changes in terms of the relative quantities of stable $\mathrm{I}^{-}$, $T_{3}$, and $T_{4}$ by the methodology presented in this study.

One of the most intriguing results observed using this technique was the daily cyclical pattern of thyroidal iodine release found in euthyroid subjects. This diurnal pattern was not present in hyperthyroidism due to either Graves' disease, toxic nodular goiter, or primary or secondary hypothyroidism. $\mathrm{T}_{3}$ administration to euthyroid subjects resulted in an abrupt disappearance of cyclicity indicating that, in part, this cyclicity was dependent on TSH stimulation. Although some alternative explanation could be proposed, the most likely factor responsible for this cyclicity would appear to be a fluctuation in TSH secretion. Utiger, employing an immunoassay for human plasma TSH, was unable to detect any diurnal variation in plasma TSH values in normal subjects (27). In contrast, Lemarchand-Beraud and Vannotti, using a bovine TSH assay system which cross-reacted with human TSH, found a diurnal trend in plasma TSH values (28). They observed the peak $\mathrm{TSH}$ values to occur at 4:00 a.m. and the nadir values at 4:00 p.m. daily. These values closely correspond to our mean peak values of 4:00 a.m. and the nadir values of 5:00 p.m. In addition, diurnal variations in pituitary or serum TSH levels have been reported to occur in laboratory animals $(7,28-30)$.

Approximately 1 wk was required in euthyroid subjects for adequate isotopic equilibration to occur such that a predictable linear rise in ${ }^{100} \mathrm{I} / \mathrm{I}$ I ratio values was achieved. During the $1 \mathrm{st}$ wk by contrast, there was a general decline in the ${ }^{120} \mathrm{I} /{ }^{20} \mathrm{I}$ ratio values. During the excretory phase, the continued dissipation of I used 
to endogenously label the thyroid gland was primarily responsible for the rapid decline. In the transition phase which followed, the continued decline in the ratio values could only be explained by the changing nature of the labeled thyroid release products. If the relative proportions of endogenously labeled release products (i.e., $T_{2}$, $T_{\text {, }}$ and $\mathrm{I}$ ) were constant, then it would be expected that the ratio values should gradually climb but never consistently fall as was seen during this phase.. A most likely explanation would be that the proportion of iodinated release products varied with time as a reflection of the heterogeneity present in the recently formed and recently mobilized labeled thyroglobulins (31). This would indicate a relative predominance of short-lived release products such as $\mathrm{I}^{-}$, iodotyrosines, and $T_{8}$ relative to $T$. during the early portion of the transition phase. A possible alternative explanation for this phenomenon would be a very rapid decline in specific activity of thyroid release products having a short biological half-life, such as $\mathrm{I}^{-}$. The elucidation of which of these explanations is correct must await further investigation.

An additional benefit of employing a ${ }^{121}$ reference indicator was that the thyroid release pattern could be studied in the unblocked thyroid gland without concern for recycling the labeled $\mathrm{I}^{-}$to the thyroid gland. Verification of this statement was provided by the observation that perchlorate, which competes with the thyroid trapping of $\mathrm{I}^{-}$, produced no significant alterations in release patterns. The use of the unblocked thyroid gland is particularly useful in evaluating the effects of antithyroid drugs such as methimazole and large amounts of potassium $\mathrm{I}^{-}$on thyroid function, particularly in regard to the efficiency with which $\mathrm{I}^{-}$is recycled into new hormone formation from the second $\mathrm{I}^{-}$pool.

A major application of this technique will be to ascertain what factors may or may not alter thyroid function. Changes, either in peripheral hormone metabolism, hypothalamic or pituitary function, or thyroid glandular activity, will be reflected by this technique since a perturbation in any one of these spheres must eventually be expressed by an alteration in thyroid glandular release. As to whether the fluctuations observed in thyroid function play an important physiological role in peripheral hormonal action cannot be determined from the data presented in this study; however, it should be stated that the potential for rapid fluctuations in thyroidal iodine release does exist in adult euthyroid subjects. To underscore this statement, a release study in a patient subjected to an attack of angina pectoris and a severe bout of Asian flu was included in this study. Marked spontaneous fluctuations in thyroid release patterns of an inhibitory nature were seen to occur coincident with these illnesses. Thus, it appears that this technique allows the detection of influences on thyroidal iodine release which have not been previously appreciated when employing other means for measuring thyroid function.

\section{ACKNOWLEDGMENTS}

I wish to express my gratitude to Miss Evangelyn Wise and Mr. Dwight W. Warren for their excellent technical assistance and to Mrs. Anne Santo for assistance in preparing the manuscript.

This study was supported, in part, by U. S. Public Health Service Grant No. AM 11727 and General Clinical Research Center Grant No. RR-43.

\section{REFERENCES}

1. Brown-Grant, K. 1966. The control of thyroid secretion. Symposium on the Thyroid Gland. J. Clin. Pathol. (London). 20 (Suppl.) : 327.

2. Harris, G. W., and J. W. Woods. 1957. Hypothalamuspituitary-thyroid relationships. Ciba Found. Colloq. on Endocrinol. 10: 3.

3. Brown-Grant, K., G. W. Harris, and S. Reichlin. 1954. The influence of the adrenal cortex on thyroid activity in the rabbit. J. Physiol. (London). 126: 41.

4. Brown-Grant, K., and G. Pethes. 1960. The response of the thyroid gland of the guinea-pig to stress. $J$. Physiol. (London). 151: 40.

5. Andersson, B., L. Ekman, C. C. Gale, and J. W. Sundsten. 1963. Control of thyrotrophic hormone (TSH) secretion by the "heat loss center." Acta Physiol. Scand. 59: 12.

6. Jobin, M., and C. Fortier. 1965. Pituitary-thyroid-adrenocortical interactions during cold exposure in the rat. Fed. Proc. 24: 149.

7. Ducommun, P., E. Sakiz, and R. Guillemin. 1966. Dissociation of the acute secretions of thyrotropin and adrenocorticotropin. Amer. J. Physiol. 210: 1257.

8. Purves, H. D. 1964. Control of thyroid function. In The Thyroid Gland. R. Pitt-Rivers and W. R. Trotter, editors. Butterworth, Co. Ltd. London. 2: 1.

9. Rall, J. E., J. Robbins, and C. G. Lewallen. 1964. The thyroid. In The Hormones. G. Pincus, K. V. Thimann and E. B. Astwood, editors. Academic Press, Inc., New York. 5: 159 .

10. Fore, W., P. Kohler, and J. Wynn. 1966. Rapid redistribution of serum thyroxine during ether anesthesia. J. Clin. Endocrinol. Metab. 26: 821.

11. Suzuki, M., T. Tonoue, S. Matsuzaki, and K. Yamamoto. 1967. Initial response of human thyroid, adrenal cortex, and adrenal medulla to acute cold exposure. Can. J. Physiol. Pharmacol. 45 : 423.

12. Irvine, C. H. G. 1968. Effect of exercise on thyroxine degradation in athletes and non-athletes. J. Clin. Endocrinol. Metab. 28: 942.

13. Charters, A. C., W. D. Odell, and J. C. Thompson. 1969. Anterior pituitary function during surgical stress and convalescence. Radioimmunoassay measurement of blood TSH, LH, FSH, and growth hormone. J. Clin. Endocrinol. Metab. 29: 63.

14. Hays, M. T., and D. H. Solomon. 1969. Effect of epinephrine on the peripheral metabolism of thyroxine. $J$. Clin. Invest. 48: 1114.

15. Ingbar, S. H. 1960. Clinical and physiologic implications of thyroxine turnover in man. In Clinical Endo- 
crinology. I. E. B. Astwood, editor. Grune \& Stratton, Inc., New York. 91.

16. Nicoloff, J. T. 1970. A new method for the measurement of acute alterations in thyroxine deiodination rate in man. J. Clin. Invest. 49: 267.

17. Nicoloff, J. T.; and J. T. Dowling. 1968. Estimation of thyroxine distribution in man. J. Clin. Invest. 47: 26.

18. Stanbury, J. B., A. A. H. Kassenaar, and J. W. A. Meijer. 1956. The metabolism of iodotyrosines. I. The fate of mono- and diiodotyrosine in normal subjects and and in patients with various diseases. J. Clin. Endocrinol. Metab. 16: 735.

19. Isaacs, G. H., J. C. Athans, and I. N. Rosenberg. 1966. Effects of thyrotropin upon thyroidal iodide; studies using thyroid venous cannulation and two radiodine isotopes. J. Clin. Invest. $45: 758$.

20. Ohtaki, S., S. Moriya, H. Suzuki, and Y. Horiuchi. 1967. Nonhormonal iodine escape from the normal and abnormal thyroid glands. J. Clin. Endocrinol. Metab. 27: 728.

21. Yamamoto, Y., S. Moriya, and Y. Horiuchi. 1969. Iodide outflow from thyroid: its relation to plasma inorganic iodide concentration. J. Clin. Endocrinol. Metab. 29: 265.

22. Nagataki, S., K. Shizume, and K. Nakao. 1969. Thyroid function in chronic excess iodide ingestion: release of inorganic iodide from thyroid gland in response to chronic iodide administration. Endocrinology. 85: 899.
23. Ingbar, S. H., and V. A. Galton. 1963. Thyroid. Ann. Rev. Physiol. 15: 361.

24. Wolff, J. 1969. Iodide goiter and the pharmacologic effects of excess iodide. Amer. J. Med. 47: 101.

25. Greer, M. A., Y. Grimm, and K. Inoue. 1969. Fate of iodide derived from intrathyroidal hydrolysis of thyroglobulin. Endocrinology. 85: 837.

26. Buhler, U. K., and L. J. DeGroot. 1969. Effect of stable iodine on thyroid iodine release. J. Clin. Endocrinol. Metab. 29: 1546.

27. Utiger, R. D. 1965. Radioimmunoassay of human plasma thyrotropin. J. Clin. Invest. 44: 1277.

28. Lemarchand-Beraud, Th., and A. Vannotti. 1969. Relationships between blood thyrotrophin level, protein bound iodine and free thyroxine concentration in man under normal physiological conditions. Acta Endocrinol. $60: 315$.

29. Bakke, J. L., and N. Lawrence. 1965. Circadian periodicity in thyroid stimulating hormone titre in the rat hypophysis and serum. Metab. (Clin. Exp.) 14: 841.

30. Singh, D. V., J. N. Panda, R. R. Anderson, and C. W. Turner. 1967. Diurnal variation of plasma and pituitary thyrotropin (TSH) of rats. Proc. Soc. Exp. Biol. Med. 126: 553 .

31. Schneider, P. B. 1964. Thyroidal iodine heterogeneity: "last come, first served" system of iodine turnover. Endocrinology. 74: 973. 